\title{
EFECTO DE LAS EXPERIENCIAS ADVERSAS DURANTE LA NIÑEZ SOBRE LA ACTIVIDAD ELECTROENCEFALOGRÁFICA EN REPOSO: UNA REVISIÓN SISTEMÁTICA
}

\author{
EFFECT OF ADVERSE CHILDHOOD EXPERIENCES ON RESTING \\ ELECTROENCEPHALOGRAPHY: A SYSTEMATIC REVIEW
}

\author{
Liliana CAmila SERENO TORRES ${ }^{1}$ Y SANDRA Milena CAMElo RoA ${ }^{2}$ \\ UNIVERSIDAD DE SAN BUENAVENTURA, SEDE BOGOTÁ
}

FECHA RECEPCIÓN: 02/08/2020 • FECHA ACEPTACIÓN: 15/10/2020

Para citar este artículo: Sereno, L., \& Camelo, S. (2020). Efecto de las experiencias adversas durante la niñez sobre la actividad electroencefalográfica en reposo: Una revisión sistemática. Psychologia, 14(2), 81-94. https://doi.org/ 10.21500/19002386.4888

\section{Resumen}

El progresivo interés en estudiar los efectos negativos en la fisiología y estructura cerebral producto de las experiencias adversas durante la niñez (EAN) ha propiciado el análisis de registros psicofisiológicos por medio de la electroencefalografía (EEG) en estados de reposo. Por tanto, el objetivo de este estudio permitió realizar una revisión sistemática basada en la metodología PRISMA para identificar las investigaciones adelantadas en los últimos 30 años. Para ello se utilizaron cuatro bases de datos: Scopus, PubMed, EBSCO y Redalyc; y dos listas de términos referentes a los tipos de EAN prevalentes en Colombia y al EEG en reposo. Inicialmente, se encontraron 21556 artículos que contaron con los términos de búsqueda; se excluyeron 21545 estudios que no cumplían con los criterios, y, finalmente, se seleccionaron 11 artículos de texto completo. Los resultados sugieren que la actividad de las bandas alfa $\alpha$ frontales derechas predicen mayor respuesta emocional a estímulos negativos, y mayor probabilidad de presentar problemas afectivos; contrario a la actividad electroencefalográfica en estas mismas bandas en regiones frontales izquierdas. Se espera que este estudio promueva investigaciones relacionadas con el análisis de perfiles electroencefalográficos y favorezca el avance de procesos terapéuticos más precisos y eficaces en la intervención del trauma.

Palabras clave: Experiencia Adversa; Niñez; Electroencefalografía en Reposo

lcserenot@academia.usbbog.edu.co, licaseto01@hotmail.com

2 smcamelo@usbbog.edu.co,psi.coordinvestigaciones@usbbog.edu.co 


\begin{abstract}
The progressive interest in studying the negative effects on brain physiology and structure produced by Adverse Experiences during Childhood (AE), has led to the analysis of psychophysiological records by means of Electroencephalography (EEG) in resting states. Therefore, the objective of this study allowed a systematic review based on the PRISMA methodology to identify the research carried out in the last 30 years. Four databases were used for this purpose: Scopus, PubMed, EBSCO and Redalyc; and two lists of terms referring to the types of EANs prevalent in Colombia and to the EEG at rest. Initially 21,556 articles were found that had the search terms, 21,545 studies were excluded that did not meet the criteria, and finally 11 full text articles were selected. Results suggest that right frontal alpha band activity $\alpha$ predicts greater emotional response to negative stimuli, and greater likelihood of presenting affective problems; contrary to electroencephalographic activity in these same bands in left frontal regions. It is expected that this study will promote research related to the analysis of electroencephalographic profiles and will favour the advancement of more precise and effective therapeutic processes in trauma intervention.
\end{abstract}

Keywords: Adverse Experience; Childhood; Resting Electroencephalography.

\section{Introducción}

Según los reportes de entidades gubernamentales como el Instituto Colombiano de Bienestar Familiar (ICBF) y el Instituto Nacional de Medicina Legal y Ciencias Forenses (INMLCF), son críticas las cifras de casos de niños, niñas y adolescentes que han vivenciado experiencias adversas durante la infancia (Instituto Colombiano de Bienestar Familiar, 2014; Instituto Nacional de Medicina Legal y Ciencias Forenses, 2019). Además, se ha documentado que la exposición a estos eventos puede ocasionar cambios significativos en el desarrollo socioemocional, los cuales se asocian con alteraciones en la estructura y fisiología cerebral (Da Silva-Ferreira, Crippa \& De Lima-Osorio, 2014). Por consiguiente, estudios recientes han utilizado instrumentos de registro psicofisiológico (PandoOrellana, Vera-García \& Lecumberri-Salazar, 2013), entre ellos, la electroencefalografía en reposo, que registra la actividad electroquímica y cortical de los perfiles de frecuencia en estados basales del cerebro (Brust-Carmona et al., 2013). De esta manera, a partir de la revisión de las investigaciones realizadas, surge la posibilidad de analizar resultados empírico-analíticos acerca de cómo las experiencias adversas durante la niñez pueden afectar la electroencefalografía en reposo, así como su asociación con la salud y los factores que pueden mediar en el desarrollo cerebral y el funcionamiento socioemocional.
En tal sentido, las experiencias adversas durante la niñez (EAN) se han definido como eventos perjudiciales, crónicos y recurrentes, con efectos acumulativos y consecuencias graves para la salud (Navalta, McGee \& Underwood, 2018). Se caracterizan por ser experiencias traumáticas que pueden ser de tipo sexual, físico o emocional, así como circunstancias familiares adversas presentadas durante la infancia o la adolescencia (Gomis \& Villanueva, 2020). Estos sucesos contribuyen al desarrollo de factores de riesgo, y hacen parte de las causas básicas de morbilidad y mortalidad en la vida adulta (Felitti et al., 1998). En línea con estos planteamientos, la Organización Mundial de la Salud (2013), en el Plan de Acción sobre salud mental 2013-2020, señaló que "la exposición a las adversidades a edades tempranas es un factor de riesgo prevenible bien establecido de los trastornos mentales" (párr. 9).

Dentro de las diferentes formas de EAN, se distinguen el descuido o la negligencia, el abuso sexual, la violencia física, emocional o psicológica, el maltrato a la madre, el abuso de sustancias psicoactivas en el hogar, enfermedades mentales en la familia, separación o divorcio de los padres, encarcelamiento de algunos de los miembros del hogar (Felitti, et al., 1998; Mesa-Gresa \& Moya-Albiol, 2011), ser testigo de violencia, tener un vecindario inseguro, institucionalización (McLaughlin et al., 2011; Cronholm et al., 2015), bajo nivel socioeconómico, victimización por pares (Finkelhor et al., 2015), discriminación, y ausencia prolongada de los 
padres (Zare et al., 2018). En Colombia, los sucesos adversos acaecidos a temprana edad que más prevalecen están relacionadas con violencia intrafamiliar, abandono, violencia sexual, pobreza extrema y situaciones de conflicto armado (Instituto Colombiano de Bienestar Familiar, 2014; Instituto Nacional de Medicina Legal y Ciencias Forenses, 2019).

Estas experiencias se asocian con exposición crónica a estresores en la infancia y resultados negativos en la salud a largo plazo (Bucci et al., 2016; Navalta, McGee \& Underwood, 2018). Algunas de estas afectaciones incluyen la obesidad, la diabetes, afecciones cardiovasculares, cáncer, enfermedades pulmonares crónicas, enfermedades hepáticas, trastornos del sueño, trastornos autoinmunes e inflamatorios (Felitti et al., 1998), síndromes metabólicos, alergias, trastornos cognitivos, trastornos mentales (Bucci, Silvério, Oh \& Burke, 2016) y de comportamiento (Sterley, Howells \& Rusell, 2011), predisposición a desarrollar trastorno bipolar (Brietzke et al., 2012), depresión, ansiedad (Zare et al., 2018), esquizofrenia (Benedetti et al., 2011), participación en conductas sexuales de riesgo (Noll, Shenk \& Putnam, 2009), impulsividad, conducta suicida (Thompson, Kingree \& Lamis, 2019) y uso de sustancias psicoactivas (Brietzke et al., 2012).

Sin duda, la exposición del cerebro en desarrollo a la respuesta al estrés ocasiona deterioro en múltiples estructuras y funciones cerebrales (Teicher \& Samson, 2016; Lackner et al., 2018; Navalta, McGee \& Underwood, 2018), cambios biológicos significativos y alteración en los sistemas alostáticos (Danese \& McEwen, 2012). Estos últimos sistemas se relacionan con el estrés y con la respuesta normal del organismo a los factores externos, internos y psicológicos que modifican el equilibrio homeostático para la sobrevivencia (De Nicola, 2015); son controlados por el sistema nervioso central (SNC), que ejerce tareas de coordinación sobre los sistemas autónomo, endocrino e inmune, junto con mediadores químicos, siendo el eje hipotalámico-hipofisario suprarrenal (HHA) el principal efector de la respuesta al estrés (McEwen \& Gianaros, 2011). Una vez desaparece el estresor, el organismo retoma su estado fisiológico basal (Lemos, 2015), pero si no logra el nivel de homeostasis, se produce desgaste o agotamiento de los sistemas alostáticos (Pilnik, 2010), generando cambios adversos multisistémicos y envejecimiento biológico (Pilnik, 2010; Danese \& McEwen, 2012).

Los efectos adversos en el organismo producto de la exposición crónica al estrés en la infancia han propiciado estudios relacionados con la afectación de las estructuras cerebrales. Entre ellos, los estudios en niños y adolescentes con historia adversa mostraron reducción del volumen cortical en el área frontal derecha (Carrion et al., 2001), en la región orbitofrontal y en el lóbulo temporal derecho (De Brito et al., 2013). Asimismo, el estrés crónico causaría en la niñez temprana hiperplasia en la amígdala e hipoplasia durante la niñez intermedia y en la adolescencia temprana (Hanson et al., 2015); además, se evidenció afectación en las zonas prefrontal media e hipocampo de adolescentes expuestos a adversidades (Lackner et al., 2018). Por su parte, en adultos que vivenciaron adversidad en la niñez, se observaron alteraciones en la materia gris y blanca, con reducción del volumen cortical (Carrion et al., 2001; Mesa-Gresa \& Moya-Albiol, 2011; Brietzke et al., 2012). Se precisa que las áreas que más han reportado cambios morfométricos en las diferentes etapas del desarrollo humano son las que están vinculadas con el procesamiento emocional, entre ellas, el hipocampo, la amígdala, la corteza prefrontal, el lóbulo temporal y el vermis cerebelar (Teicher \& Samson, 2016).

Los estudios sobre las afectaciones cerebrales producto del trauma infantil han utilizado instrumentos de registro psicofisiológico para favorecer la identificación de marcadores neurobiológicos (Pando-Orellana, VeraGarcía \& Lecumberri-Salazar, 2013); una de estas técnicas es la electroencefalografía, que resulta ser uno de los procedimientos de apoyo diagnóstico más utilizados por no generar ningún riesgo al paciente (Ramos-Argüelles et al., 2009; Brust-Carmona et al., 2013) y facilita la predictibilidad de las oscilaciones cerebrales que subyacen tras las señales observadas (Fernández et al., 2010). Este instrumento evalúa la superficie craneal para observar los registros de la actividad eléctrica funcional de conjuntos de neuronas (Ramos-Argüelles et al., 2009), permitiendo identificar registros en el cuero cabelludo derivados de potenciales postsinápticos de la actividad sincronizada de las neuronas corticales dispuestas en capas, con sus dendritas apicales perpendiculares a la superficie de la corteza (Treviño \& Gutiérrez, 2007) 
El registro de la actividad eléctrica cortical captada por el EEG se compone de ritmos, que corresponden a ondas que aparecen aisladas o en grupo, diferenciadas por parámetros de frecuencia, distribución topográfica, reactividad, forma, amplitud y duración (Talamillo, 2011). Los tipos de ritmos cerebrales más reconocidos son delta $\delta(1-3 \mathrm{~Hz})$, theta $\theta(4-7 \mathrm{~Hz})$, alfa $\alpha(8-13$ $\mathrm{Hz})$, beta $\beta(14-30 \mathrm{~Hz})$ (Ricardo \& Rueda, 2009), gamma $\gamma(>30 \mathrm{~Hz})$ (Mauriera, 2018), y el ritmo Mu, que es un subgrupo del ritmo alfa $\alpha(9-11 \mathrm{~Hz}$ ) (De Bruin, Van del Zwan \& Bögels, 2016). Las bandas delta $\delta$ y theta $\theta$ se distinguen por tratarse de actividades electroencefalográficas lentas, mientras que las bandas alfa $\alpha$ y beta $\beta$ se asocian a actividades electroencefalográficas rápidas (Ricardo \& Rueda, 2009). Para el estudio de estas ondas, se ha sugerido el análisis de patrones de la actividad electroquímica y cortical de los perfiles de frecuencia, especialmente en estados de reposo asociados a menor estimulación ambiental (Brust-Carmona et al., 2013). Algunos autores han presumido mejor relación entre el trauma infantil y alteraciones en la excitación cortical durante la realización de una tarea que requiriera atención sostenida e inhibición del comportamiento (Howells, Stein \& Russell, 2012).

Por medio de la electroencefalografía en estados de reposo, se han realizado investigaciones en muestras de personas que han presentado historia adversa en la infancia. Es así como en adultos no clínicos con reportes de maltrato infantil graves se encontró asociación positiva entre la asimetría del EEG prefrontal del lado derecho y procesos inflamatorios de bajo grado, siendo un factor de vulnerabilidad para desarrollar trastornos afectivos (Hostinar et al., 2017). En un estudio realizado en adultos con historia de trauma infantil, que se organizó en dos fases, la primera evaluó registros corticales del EEG en reposo y la segunda durante la realización de tareas visuales go/no go (GNG), los resultados mostraron correlación entre la negligencia física y emocional con la disminución de la potencia de banda alfa $\alpha$ tónica parietal izquierda durante las condiciones de reposo y durante la tarea GNG, señalando así evidencia de que el tipo de trauma infantil está asociado con una excitación cortical alterada y con la forma de experiencia adversa vivida en la infancia (Howells, Stein \& Russell, 2012; Jin et al., 2018). Por su parte, en muestras de mujeres con antecedentes de maltrato infantil se denotó mayor actividad en el EEG en las bandas alfa $\alpha$ y reducción interhemisférica frontal anterior, patrones asociados con abuso y negligencia (Miskovic, et al., 2010); a su vez, mujeres adolescentes con antecedentes similares presentaron actividad en las bandas alfa $\alpha$ frontales (Tang et al., 2018).

En los estudios llevados a cabo en niños que contaban con madres que mostraban afecto negativo se observó mayor asimetría en la banda alfa $\alpha$ frontal izquierda (Brooker, Davidson \& Goldsmith, 2016); y en niños institucionalizados desde los 23 meses hasta 54 meses de edad se registró aumento en la actividad de la banda alfa $\alpha$ frontal derecha y activación brusca frontal izquierda en la misma banda (McLaughlin et al., 2011). En una investigación longitudinal en niños evaluados desde los dos hasta los ocho años que contaban con intervención psicosocial por maltrato intrafamiliar se identificaron retrasos corticales e inmadurez en la infancia media, y en el registro electroencefalográfico, una potencia espectral de alta frecuencia mejorada en la banda beta $\beta$ (12-20Hz) (Bick et al., 2019).

En otros trabajos realizados en muestras de adolescentes con historia de maltrato infantil, la asimetría en el EEG de la banda alfa $\alpha$ frontal derecha se asoció positivamente con los síntomas del trauma y conductas problemáticas, resultados contrarios en la asimetría de la banda alfa $\alpha$ frontal izquierda (Miskovic et al., 2009). Asimismo, en adolescentes con historia adversa, se correlacionó la vulnerabilidad socioemocional (Lahat et al., 2018) y comportamientos problemáticos (Meiers et al., 2020), denotando mayor actividad de la banda alfa $\alpha$ frontal izquierda en los registros del EEG (Hassan et al., 2019). Apoyando estos resultados, en tres metaanálisis, que incluyeron 38 investigaciones, se concluyó que los factores de riesgo psicosocial en la infancia estarían relacionados con mayor actividad del registro en el EEG en regiones prefrontales derechas, especialmente en adolescentes mujeres (Peltola et al., 2014); y en una revisión sistemática, se encontró como principal hallazgo la asociación entre la asimetría de la banda alfa $\alpha$ frontal y síntomas de TEPT en adultos con historia adversa (Lobo et al., 2015).

Los hallazgos espectrales, descritos anteriormente, se orientan a apoyar planteamientos sobre lateralidad cerebro emoción, tal como precisa la hipótesis de valencia de los estímulos (Davidson \& Irwin, 1999; Murphy, 
Nimmo-Smith \& Lawrence, 2003); esta sustenta el reconocimiento y la regulación emocional como actividad bilateral, en donde el hemisferio derecho se especializa en el procesamiento de emociones negativas, mientras que el hemisferio izquierdo se asocia al procesamiento de emociones positivas (Ahern \& Schwartz, 1985; Torro, Fukusima \& Aznar-Casanova, 2008; Gainotti, 2019).

\section{Método}

Tipo de estudio

Se realizó una investigación de tipo documental a través de una revisión sistemática como diseño de investigación observacional retrospectivo, basado en la metodología PRISMA (Preferred Reporting Items for Systematic Reviews and Meta-Analyses). Esta orientación metodológica, promulgada en 2009, actualizó la declaración de QUOROM (Quality Of Reporting Of Metaanalysis) de 1999, para establecer estándares y normas de publicación dirigidos a la presentación de revisiones sistemáticas y metaanálisis, permitiendo sintetizar resultados de múltiples investigaciones primarias (Urrútia \& Bonfill, 2010; Panic et al., 2013).

\section{Unidad de análisis}

Artículos publicados entre enero de 1990 y abril de 2020 en las bases de datos Scopus, PubMed, EBSCO y Redalyc; fuentes bibliográficas en inglés y en español, respectivamente.

\section{Criterios de inclusión}

Artículos de investigación de corte empírico analítico, de texto completo en inglés y en español, publicados entre enero de 1990 y abril del 2020. Estudios que incluyeran muestras de personas no clínicas, de varias edades, ambos sexos, diferentes orientaciones de género y distinta nacionalidad, que vivenciaron los tipos de EAN elegidas por ser prevalentes en Colombia (abuso emocional, físico sexual, maltrato físico y psicológico, pobreza, adopción, violencia doméstica, conflicto armado, negligencia e inseguridad), asociados con términos del EEG en reposo (electroencefalografía, EEG, electroencefalografía en reposo, EEG en reposo, ritmos alfa, beta, delta y theta), palabras corroboradas en thesaurus y buscadas en los dos idiomas mencionados.
Criterios de exclusión

Estudios conceptuales, revisiones sistemáticas, metaanálisis, simposios, congresos y publicaciones que no incluyan el EEG en reposo como instrumento de medición asociado a los tipos de EAN seleccionados para el estudio, o que incluyan otros tipos de EAN como la victimización por pares, abandono, institucionalización y enfermedad, debido a que no cuentan con literatura suficiente y no se encuentran dentro de las experiencias adversas prevalentes en Colombia.

\section{Instrumentos}

\section{Motores de búsqueda}

Scopus ${ }^{\circledR}$ : incluye referencias bibliográficas y citas de la producción de investigación científica del mundo en todas las disciplinas. Indexa el contenido de 24600 títulos activos y 5000 editores seleccionados por un comité de revisión independiente (Elsevier, 2020).

PubMed: base de datos desarrollada por el Centro Nacional de Información Biotecnológica (NCBI) y la Biblioteca Nacional de Medicina (NLM). Comprende más de 30 millones de citas de literatura biomédica de MEDLINE, principalmente de las áreas de biomedicina y salud, y disciplinas relacionadas con las ciencias de la salud, del comportamiento, químicas y bioingeniería (NLM, 1996).

EBSCO: proveedor de bases de datos de investigación, revistas electrónicas, suscripciones a revistas, libros electrónicos y servicios de descubrimiento para bibliotecas de todo tipo (EBSCO, 2020).

Redalyc: Red de Revistas Científicas de América Latina y el Caribe, España y Portugal, que integra revistas de alta calidad científica y editoriales de la región. En 2019 alcanzó 1310 revistas, 50000 fascículos y 650000 artículos (Redalyc, 2019).

\section{Procedimiento}

La presente revisión sistemática, basada en las directrices de la metodología PRISMA, utilizó el diagrama de flujo de información de cuatro fases (Figura 1). 


\section{Fase uno}

Identificación

Selección de 21556 artículos en título, resumen y palabras clave en las bases de datos mencionadas, teniendo en cuenta la construcción de dos listas de palabras relacionadas entre sí, y corroboradas en thesaurus. La primera lista se conformó con palabras clave y los tipos de EAN que más prevalecen en Colombia; la segunda lista, palabras relacionadas con la electroencefalografía en reposo.

\section{Fase dos}

Tamizaje

Se descartaron 11720 citas correspondientes a estudios duplicados, conceptuales o que no incluían el EEG en reposo como instrumento de medición correla- cionado con las EAN elegidas; se seleccionaron 108 artículos para evaluar el resumen o abstract.

Fase tres

Elegibilidad

De los 108 estudios seleccionados en la fase anterior, se excluyeron 42 artículos que no cumplían con las variables establecidas, quedando 66 para evaluar el texto completo.

\section{Fase cuatro}

Inclusión

De los 66 artículos seleccionados en la fase anterior, se excluyeron 55 estudios por no cumplir con los criterios establecidos. Finalmente, fueron seleccionados 11 estudios de texto completo.

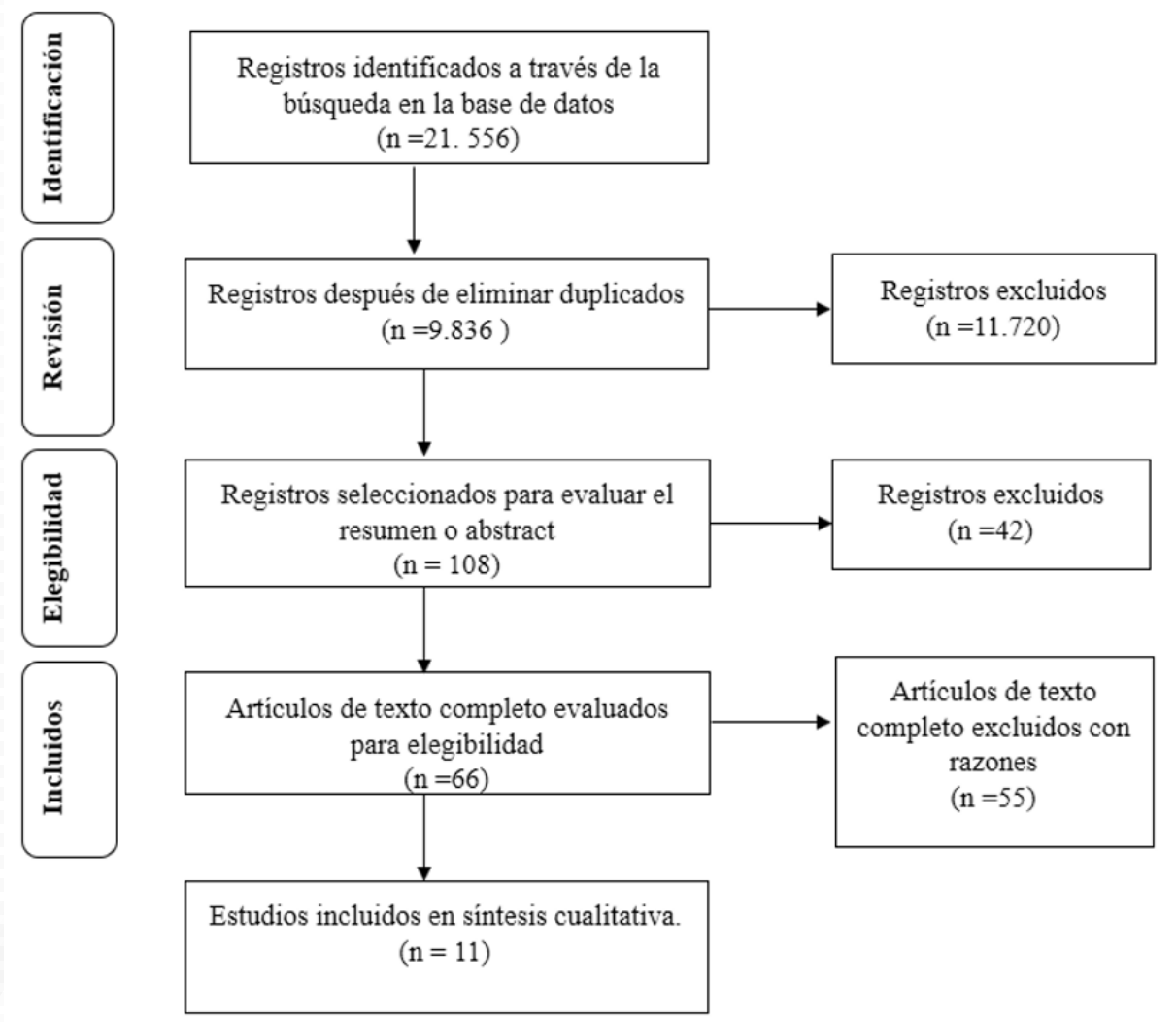

Figura 1. Diagrama de Flujo PRISMA (2009)

\section{Resultados}

En la Tabla 1 se muestran los once artículos elegidos para la revisión encontrados en la base de datos
PubMed; la publicación más antigua data del 2007 y la más reciente del 2020. Dentro de las características metodológicas, cuatro estudios utilizaron diseño y tipo de investigación retrospectivo transversal y siete artículos 
implementaron diseño prospectivo longitudinal (Figura 2). En los trabajos se eligieron experiencias adversas referentes al abuso emocional, físico, sexual, negligencia física y emocional; ocho estudios seleccionaron muestras de personas que experimentaron maltrato en la infancia; a su vez, no se identificaron investigaciones que incluyeran las demás experiencias adversas prevalentes en nuestro país seleccionadas para la revisión, entre ellas, pobreza, adopción, violencia doméstica, conflicto armado e inseguridad. Asimismo, los estudios revisados eligieron participantes no clínicos de varias nacionalidades; cuatro investigaciones contaron con participantes estadounidenses, cuatro artículos incluyeron individuos canadienses, dos eligieron participantes sur coreanos y uno contó con participación de individuos sudafricanos. Con referencia al sexo y etapa de desarrollo, cinco trabajos seleccionaron participantes adolescentes mujeres; dos artículos evaluaron niños de ambos sexos entre los dos y los 12 años; tres estudios contaron con adultos de ambos sexos entre los 27 y los 30 años, y en una muestra de adultos de ambos sexos se identificó una edad media de 55,3. Los estudios realizados en adolescentes incluyeron únicamente muestras de mujeres que habían experimentado maltrato infantil, y su diseño de investigación fue de tipo prospectivo longitudinal (Figura 2).

\section{Tabla 1}

Artículos de texto completo seleccionados para la revisión

\begin{tabular}{l}
\hline \hline Título \\
\hline Alpha EEG asymmetry, childhood maltreatment, and problem behaviors: A pilot \\
home-based study
\end{tabular}

Psychophysiological influences on personality trajectories in adolescent females exposed to child maltreatment Psychophysiological influences on personality trajectories in adolescent females exposed to child maltreatment

An Integrated Model of Emotional Problems, Beta Power of Electroencephalography, and Low Frequency of Heart Rate Variability after Childhood Trauma in a Non-Clinical Sample: A Path Analysis Study

Longitudinal Associations Among Child Maltreatment, Resting Frontal Electroencephalogram Asymmetry, and Adolescent Shyness

Early Parenting Intervention and Adverse Family Environments Affect Neural Function in Middle Childhood.

Trajectories of resting frontal brain activity and psychopathology in female adolescents exposed to child maltreatment

Frontal brain asymmetry, childhood maltreatment, and low-gradeinflammation at midlife

Childhood Trauma Associated with Enhanced High Frequency Band Powers and Induced Subjective Inattention of Adults

Childhood trauma is associated with altered cortical arousal: insights from an EEG study

Stability of Resting Frontal Electroencephalogram (EEG) Asymmetry and Cardiac Vagal Tone in Adolescent Females Exposed to Child Maltreatment

Emotion and resilience: A multilevel investigation of hemispheric electroencephalogram asymmetry and emotion regulation in maltreated and nonmaltreated children

\section{Autor y año de publicación}

Meiers, Nooner, De Bellis, Debnath y Tang (2020)

Hassan, MacMillan, Tanaka y Schmidt (2019)

Jin, Kim, Kim, Hyun y Lee (2018)

Lahat, Tang, Tanaka, Van

Lieshout, MacMillan y Schmidt (2018)

Bick, Palmwood, Zajac, Simons y

Dozier (2018)

Tang, Miskovic, Lahat, Tanaka, MacMillan, Van Lieshout y Schmidt (2018)

Hostinar, Davidson, Graham, Mroczek, Lachman, Seeman, Van Reekumf y Miller (2017)

Lee, Park, Jin, Lee y Hahn (2017)

Howells, Stein y Russell (2012)

Miskovic, Schmidt, Georgiades, Boyle y MacMillan (2009)

Curtis y Cicchetti (2007) 


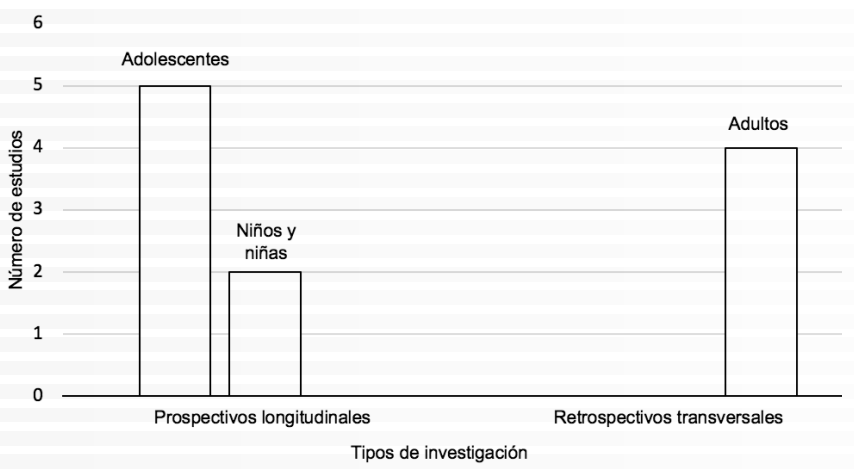

Figura 2. Tipo de investigación y muestras incluidas en los artículos elegidos.

Con referencia a los instrumentos implementados, se utilizó la electroencefalografía en estados de reposo, administrando previamente cuestionarios de autoinforme, siendo el Cuestionario del Trauma Infantil (CTQ) el más utilizado por los investigadores. En la aplicación del EEG se siguieron procedimientos estándares para registrar la actividad eléctrica cerebral, con la utilización de electrodos y su ubicación con la norma internacional 10/20 para evaluar los registros en las distintas regiones cerebrales. Cada estudio eligió áreas específicas para su medición, observando que dos trabajos eligieron regiones anteriores, centrales y posteriores del cerebro; cuatro estudios seleccionaron las regiones frontales, centrales, parietales y occipitales; dos eligieron regiones frontales; uno seleccionó las regiones frontales y parietales; en otro se evaluaron las regiones frontales, centrales, temporales y occipitales; otro incluyó las regiones frontales y parietales; y en uno se seleccionaron las regiones parietales. Asimismo, se evidenció que la banda alfa $\alpha$ fue incluida en el total de estudios; dos trabajos incluyeron las bandas más reconocidas (delta $\delta$, theta $\theta$, alfa $\alpha$, beta $\beta$ y gamma $\gamma$ ); dos eligieron las bandas theta $\theta$, alfa $\alpha$, y beta $\beta$; seis investigaciones seleccionaron únicamente la banda alfa $\alpha$, y un estudio no especificó las bandas.

Respecto a los trabajos con participantes adultos, se identificó que estos fueron de tipo retrospectivo transversal. Algunos de los resultados más significativos denotaron la correlación entre la actividad de la banda beta $\beta$ en regiones centrales y frontales del cerebro con la presencia de problemas emocionales, labilidad afectiva, ansiedad y depresión en la adultez (Jin et al.,
2018). En una investigación se sugirió que la actividad de las bandas delta $\delta$, beta $\beta$ y gamma $\gamma$ en las mismas regiones, comparadas con los resultados de los grupos controles, pudiesen predisponer para el desarrollo de problemas neurocognitivos incluyendo los procesos atencionales como consecuencia de historia adversa en la infancia (Lee et al., 2017). Asimismo, en el estudio realizado por Howells, Stein y Russell (2012) en adultos con historia de trauma infantil tanto en las fases de reposo como en la realización de tareas visuales go/ no go (GNG) se identificaron correlaciones entre la negligencia física y emocional con la disminución de la potencia de banda alfa $\alpha$ tónica parietal izquierda durante ambas condiciones (Howells, Stein \& Russell, 2012; Jin et al., 2018). Por su parte, en muestras símiles se encontró asociación positiva entre la asimetría del EEG prefrontal derecho y procesos inflamatorios de bajo grado como factor de vulnerabilidad para desarrollar trastornos afectivos (Hostinar et al., 2017).

Los resultados de los estudios prospectivos longitudinales realizados en adolescentes mujeres que habían experimentado maltrato concluyeron mayor actividad electroencefalográfica frontal derecha en un periodo de seis meses, sugiriendo estabilidad en la actividad del EEG; esta actividad se explicó por la reducción de la banda alfa $\alpha$ frontal derecha y aumento de la actividad de la banda alfa $\alpha$ frontal izquierda, diferencias que tienen gran importancia funcional por el sustrato único de cada patrón; es decir, que la hiperactivación frontal derecha estaría asociada con un aumento de respuestas de ansiedad; mientras que la hipoactivación frontal izquierda, con la reducción de comportamientos relacionados con síntomas anhedónicos de la depresión (Miskovic et al., 2009; Hassan et al., 2019). Por su parte, entre las adolescentes que exhibían una asimetría frontal relativa derecha, las participantes con historia de maltrato informaron mayor timidez, neuroticismo, ansiedad generalizada y menor extraversión; planteando este estudio que la actividad cerebral frontal derecha y la timidez estarían involucradas en la vulnerabilidad socioemocional de las adolescentes con historia de maltrato (Lahat et al., 2018). Asimismo, las adolescentes con asimetría alfa $\alpha$ frontal izquierda estable y niveles más bajos de trauma tuvieron menor probabilidad de presentar síntomas de TEPT y depresión durante dos 
años, que aquellas con asimetría alfa $\alpha$ frontal derecha. Estos hallazgos plantean que los patrones de desarrollo de la actividad del cerebro frontal izquierdo en reposo pudiesen amortiguar la psicopatología en mujeres jóvenes maltratadas (Tang et al., 2018), y sugieren que la asimetría frontal derecha de la banda alfa $\alpha$ estaría involucrada en la asociación positiva de los síntomas y problemas del comportamiento relacionados con el trauma (Meiers et al., 2020).

En los estudios llevados a cabo en niños, la adversidad temprana en el hogar se asoció con perfiles electroencefalográficos que indicaron retrasos corticales e inmadurez en la infancia media, basados en la actividad de las bandas de frecuencia más bajas y menor actividad en la banda alfa $\alpha$; los niños con historia de maltrato mostraron mayor actividad de frecuencia en las bandas beta $\beta$ que los niños control (Bick et al., 2019). La capacidad para regular las emociones se asoció con una actividad mayor del EEG frontal izquierdo, la asimetría en las regiones corticales centrales distinguió entre niños con mayor nivel y menor nivel de adaptación, mostrando una mayor actividad del hemisferio izquierdo en los niños más resilientes; a su vez, los niños control mostraron mayor actividad del EEG del hemisferio izquierdo en las regiones corticales parietales (Curtis \& Cicchetti, 2007).

\section{Discusión}

Por medio de una revisión sistemática, el presente estudio se enfocó en evaluar los registros psicofisiológicos observados en la electroencefalografía en estados de reposo asociados con los efectos negativos de las experiencias adversas presentadas en la infancia, permitiendo cuantificar las investigaciones realizadas en los distintos continentes y analizar sus resultados. En los hallazgos se evidenció la influencia de la actividad de la banda alfa $\alpha$ en regiones izquierdas frontales del cerebro en niños, adolescentes mujeres y en adultos, que favorecería la disminución de los efectos negativos a largo plazo en las personas con historia adversa en la infancia; mientras que la asimetría de las regiones cerebrales derechas frontales de la banda alfa $\alpha$ moderaría la asociación positiva de síntomas y problemas del comportamiento relacionados con el trauma (Meiers et al., 2020). De esta manera, la asimetría del EEG prefrontal estaría vinculada con factores de vulnerabilidad para desarrollar trastornos afectivos como depresión y trastornos de ansiedad (Hostinar et al., 2017), resaltando así, que la banda alfa $\alpha$ frontal izquierda, pudiese disminuir la presencia de psicopatologías en mujeres jóvenes (Tang et al., 2018); además, según los autores que evaluaron muestras infantiles, una mayor actividad electroencefalográfica en las regiones frontales izquierdas supondría una mejor capacidad para regular las emociones (Curtis \& Cicchetti, 2007). Estos resultados aportan a los estudios sobre el desarrollo del trauma y concuerdan con los planteamientos de Fisher (2014) sobre la implicación de las regiones frontales derechas del cerebro en la valencia y en la regulación emocional, y, a su vez, se encuentran en coherencia con los modelos duales de las emociones y los hemisferios cerebrales (Davidson \& Irwin, 1999; Murphy, Nimmo-Smith \& Lawrence, 2003).

Algunas limitaciones evidenciadas en la presente revisión dan muestra de escasos estudios sobre el efecto acumulativo de las experiencias adversas vivenciadas en etapas tempranas del desarrollo, y aún más en países como el nuestro que presenta historia de violencia y situaciones sociales desfavorables. Autores como Castillo, Cleves, García, Laverde, Medina, Cortés, Ramírez y Dávalos (2017) afirman que, a pesar de la prevalencia de eventos adversos en la niñez en Colombia, no se les ha prestado la atención debida y no se ha medido el impacto real a corto y largo plazo. En línea con estos planteamientos, Witt, Brown, Plener, Brahler y Fegert (2017) mencionan que no solo faltan análisis centrados en los efectos acumulativos de las experiencias adversas en la niñez, sino que existe limitada especificidad de los diferentes subtipos de maltrato y otras adversidades en la infancia. Por su parte Howells, Stein y Russell (2012) afirman que los traumas infantiles se asocian con problemas psicológicos, pero los mecanismos psicobiológicos que explican este vínculo no se han comprendido con exactitud.

Asimismo, en esta revisión se encontró un menor número de estudios realizados con población adulta; sin embargo, los resultados en este tipo de muestras arrojaron conclusiones significativas, relacionadas con la actividad electroencefalográfica en regiones centrales y frontales del cerebro. Tal como lo afirman Lee, Park, Jin, Lee y Hahn (2017), están asociadas con el desarrollo de problemas emocionales y neurocognitivos en la adultez 
como consecuencia de historia adversa en la niñez. Otro aspecto importante es el hecho de que la mayoría de las investigaciones trabajaron preferentemente con adolescentes mujeres, para lo que Curtis y Cicchetti (2007) refieren que en la actualidad falta conceso en la literatura acerca las relaciones entre el género y los patrones registrados en la electroencefalografía; esto, por la preferencia en la elección de las muestras que pudiese sesgar la interpretación de los resultados.

Por tanto, esta revisión es uno de los primeros trabajos realizados en Latinoamérica y en Colombia enfocados en el desarrollo del trauma y la electroencefalografía, que pone de manifiesto el estado actual de las investigaciones en el área. Para futuras investigaciones se sugiere realizar la búsqueda en otros idiomas y ampliar la cobertura con otros motores de búsqueda para obtener mayor cantidad de publicaciones relacionadas.

\section{Referencias}

Ahern, G. L., \& Schwartz. (1985). Differential lateralization for positive and negative emotion in the brain: EEG spectral analysis. Neuropsychologia, 23(6), 745-755. https://doi.org/10.1016/00283932(85)90081-8

Benedetti, F., Radaelli, D., Poletti, S., Falini, A., Cavallaro, R., Dallaspezia, S., Riccaboni, R., Scotti, G., \& Smeraldi, E. (2011). Emotional reactivity in chronic schizophrenia: structural and functional brain correlates and the influence of adverse childhood experiences. Psychological Medicine, 41(3), 509-519. https://doi.org/10.1017/ S0033291710001108

Biblioteca Nacional de Medicina. (1996). Recuperado de https://pubmed.ncbi.nlm.nih.gov/about/

Bick, J., Palmwood E. N., Zajac. L., Simons, R., \& Dozier, M. (2019). Early Parenting Intervention and Adverse Family Environments Affect Neural Function in Middle Childhood. Biological Psychiatry, 85(4), 326-335. https://doi.org/10.1016/j. biopsych.2018.09.020

Brietzke, E., Kauer Sant'anna, M., Jackowski, A., GrassiOliveira, R., Bucker, J., Zugman, A., Mansur, R. B., \& Bressan, R. A. (2012). Impact of Childhood Stress on Psychopathology. Braz J Psychia- try, 34(4), 480П488. https://doi.org/10.1016/j. rbp.2012.04.009

Brooker, R. J., Davidson, R. J., \& Goldsmith, H. H. (2016). Maternal negative affect during infancy is linked to disrupted patterns of diurnal cortisol and alpha asymmetry across contexts during childhood. Journal of Experimental Child Psychology, 142, 274-290. https://doi.org/10.1016/j. jecp.2015.08.011

Brown, E. D., Anderson, K. E., Garnett, M. L., \& Hill, E. M. (2019). Economic instability and household chaos relate to cortisol for children in poverty. Journal of Family Psychology, 33(6), 629-639. https://doi.org/10.1037/fam0000545

Brust-Carmona, H., Galicia, M., Flores, B., Borunda F., \& Yáñez, O. (2013). Las neurociencias en el diagnóstico y en la evaluación de la rehabilitación integral de secuelas de lesiones cerebrales en el INR. Investigación en discapacidad Medigraphic, 2(1), 28-37. Available at https://www.medigraphic.com/ cgi-bin/new/resumen.cgi? IDARTICULO $=40368$

Bucci, M., Silvério S., Oh, D., \& Burke, N. (2016). Toxic Stress in Children and Adolescent. Elsevier Advances in pediatrics, 63, 403-428. https://doi. org/10.1016/j.yapd.2016.04.002

Camras, L. A., Grow, J. G., \& Ribordy, S. C. (1983). Recognition of emotional expression by abused children. Journal of Clinical Child Psychology, 12(3), 325-328. https://doi.org/10.1080/15374418309533152

Carrion, V. G., Weems, C. F., Eliez, S., Patwardhan, A., Brown, W., Ray, R. D., \& Reiss, A. L. (2001). Attenuation of Frontal Asymmetry in Pediatric Posttraumatic Stress Disorder. Biol Psychiatry, 50(12), 943-951. https://doi.org/10.1016/S00063223(01)01218-5

Castillo, A., Cleves, D., García, A. M, Laverde, L., Medina, V., Cortés, H., Ramírez, D., \& Dávalos, D. M. (2017). Experiencias Adversas de la Infancia en una muestra de pacientes con enfermedad crónica en Cali-Colombia. Medicina U.P.B, 36(1), 9-15. Recuperado de https://www.redalyc.org/articulo. oa? id = 1590/159051101001

Cronholm, P. F., Forke, C. M., Wade, R., Bair-Merritt, M. H., Davis, M., Harkins-Schwarz, M., Pachter, L. M., \& Fein, J. A. (2015). Adverse Childhood Experiences: Expanding the Concept of 
Adversity. American Journal of Preventive Medicine, 49(3), 354-361. https://doi.org/10.1016/j. amepre.2015.02.001

Curtis, W. J., \& Cicchetti, D. (2007). Emotion and resilience: A multilevel investigation of hemispheric electroencephalogram asymmetry and emotion regulation in maltreated and nonmaltreated children. Development and Psychopathology, 19(3), 811-844. https://doi.org/10.1017/ S0954579407000405

Choi, J., Jeong, B., Rohan, M. L., Polcari, A. M., \& Teicher, M. H. (2009). Preliminary Evidence for White Matter Tract Abnormalities in Young Adults Exposed to Parental Verbal Abuse. Biological Psychiatry, 65(3), 227-234. https://doi.org/10.1016/j. biopsych.2008.06.022

Danese, A., \& McEwen, B. S. (2012). Adverse Childhood experiences, allostasis, allostatic load, and age-related disease. Physiology \& Behavior, 106(1), 29-39. https://doi.org/10.1016/j.physbeh.2011.08.019

Dannlowski, U., Stuhrmann, A., Beutelmann, V., Zwanzger, P., Lenzen, T., Grotegerd, D., Domschke, K., Hohoff, C., Ohrmenn, P., Bauer, J., Lindner, C., Postert, C., Konrad, C., Arolt, V., Heindel, W., Suslow, T., \& Kugel, H. (2012). Limbic Scars: Long-Term Consequences of Childhood Maltreatment Revealed by Functional and Structural Magnetic Resonance Imaging. Biological Psychiatry, 71 (4), 286-293. https://doi.org/10.1016/j.biopsych.2011.10.021

Da Silva-Ferreira, G. C., Crippa, J. A. S., \& De Lima-Osorio, F. (2014). Facial emotion processing and recognition among maltreated children: A systematic literature review. Frontiers in Psychology, 5(1460), 1-10. https://doi.org/10.3389/fpsyg.2014.01460

Davidson, R. J., \& Irwin, W. (1999). The functional neuroanatomy of emotions and affects style. Trends in Cognitive Sciences, 3(1), 11-21. https://doi. org/10.1016/s1364-6613(98)01265-0

De Bellis M., \& Zisk A. (2014). The biological effects of childhood trauma. Child Adolesc Psychiatr Clin, 23, 185-222. https://doi.org/10.1016/j. chc.2014.01.002

De Brito, S. A., Viding, E., Sebastian, C. L., Kelly, P. A., Mechelli, A., Maris, H., \& McCrory, E. J. (2013). Reduced orbitofrontal and temporal grey mat- ter in a community sample of maltreated children. Journal of Child Psychology and Psychiatry and Allied Disciplines, 54(1), 105-112. https://doi. org/10.1111/j.1469-7610.2012.02597.x

De Bruin, E., Van del Zwan, J., \& Bögels, S. (2016). A RCT comparing daily mindfulness meditations, biofeedback exercises and daily physical exercise on attention control, executive functioning, mindful awareness, self-compassion and worrying in stressed young adults. Mindfulness, 7(5), 11821192. https://doi.org/10.1007/s12671-0160561-5

De Nicola, A. F. (2015). Mecanismos neuroendocrinos de respuesta durante el estrés y la carga alostática. Ciencia e Investigación, 65(1), 17-26. Recuperado de https://ri.conicet.gov.ar/bitstream/handle/11336/3067/Ciencia_e_Investigacion_2015. pdf? sequence $=1$ \&isAllowed $=\mathrm{y}$

Elsevier. (2020). How Scopus works. Getting the most out of published research. Available at https:// www.elsevier.com/solutions/scopus/how-scopusworks

Elton Bryon Stephens Company EBSCO. (2020). About EBSCO. Available at https://www.ebsco.com/ about

Evans, G. W., Kim, P., Ting, A. H., Tesher, H. B., \& Shannis, D. (2007). Cumulative Risk, Maternal Responsiveness, and Allostatic Load Among Young Adolescents. Developmental Psychology, 43(2), 341-351. https://doi.org/10.1037/00121649.43.2.341

Felitti V. J., Anda, R. F., Nordenberg, D., Williamson, D. F., Spitz, A. M., Edwards, V., Koss, M. P., \& Marks J.S. (1998). Relationship of childhood abuse and household dysfunction to many of the leading causes of death in adults: the adverse childhood experiences (ACE) study. American Journal of Preventi Medicine, 14(4), 245-258. https://doi.org/10.1016/ s0749-3797(98)00017-8

Fernández, A., Andreina, M., Hornero, R., Ortiz, T., \& López-Ibor, J. J. (2010). Análisis de complejidad de la actividad cerebral y trastornos mentales. Actas Esp Psiquiatr, 38(4), 229-238. Recuperado de https://www.actaspsiquiatria.es/repositorio/11/64/ ESP/11-64-ESP-229-238-357613.pdf 
Finkelhor, D., Shattuck, A., Turner, H., \& Hamby, S. (2015). A revised inventory of Adverse Childhood Experiences. Child Abus Negl, 48, 13-21. https:// doi.org/10.1016/j.chiabu.2015.07.011

Fisher, S. F. (2014). Neurofeedback in the treatment of developmental trauma: calming the fear-driven brain. WW Norton \& Company.

Gainotti, G. (2019). The Role of the Right Hemisphere in Emotional and Behavioral Disorders of Patients with Frontotemporal Lobar Degeneration: An Updated Review. Frontiers in aging neuroscience, 11 (55). https://doi.org/10.3389/fnagi.2019.00055

Giraldo, E., Acosta, C. D., \& Castellanos-Domínguez, G. (2010). Estimación dinámica neuronal a partir de señales electroencefálicas sobre un modelo realista de la cabeza. Revista Tecno Lógicas, 25, 151- 167. https://doi.org/10.22430/22565337.129

Gomis-Pomares, A., \& Villanueva, L. (2020). The effect of adverse childhood experiences on deviant and altruistic behavior during emerging adulthood. Psicothema, 32(1), 33-39. https://doi.org/10.7334/ psicothema2019.142

Hanson, J. L., Nacewicz, B. M., Sutterer, M. J., Cayo, A. A., Schaefer, S. M., Rudolph, K. D., \& Davidson, R. J. (2015). Behavioral problems after early life stress: Contributions of the hippocampus and amygdala. Biological Psychiatry, 77(4), 314-323. https://doi.org/10.1016/j.biopsych.2014.04.020

Hassan, R., MacMillan, H. L., Tanaka, M., \& Schmidt, L. A. (2019). Psychophysiological influences on personality trajectories in adolescent females exposed to child maltreatment. Development and Psychopathology, 1-12. https://doi.org/10.1017/ S0954579419001342

Hostinar, C. E., Davidson, R. J., Graham, E. K., Mroczek, D. K., Lachman, M. E., Seeman, T. E., Van Reekum, C. M., \& Miller, G. E. (2017). Frontal brain asymmetry, childhood maltreatment, and lowgrade inflammation at midlife. Psychoneuroendocrinology, 75, 152-163. https://doi.org/10.1016/j. psyneuen.2016.10.026

Howells, F. M, Stein, D. J., \& Russell, V. A. (2012). Childhood Trauma is Associated with Altered Cortical Arousal: Insights from an EEG Study. Frontiers in Integrative Neuroscience, 6(120), 1-19. https://doi. org/10.3389/fnint.2012.00120
Instituto Colombiano de Bienestar Familiar. (2014). Recuperado de http://www.me.gov.ar/construccion/ pdf_derechos/maltrato_infantil_orient.pdf

Instituto Nacional de Medicina Legal y Ciencias Forenses. (2019). Recuperado de http://www.medicinalegal.gov.co/documents/20143/386932/ Forensis + 2018.pdf/be4816a4-3da3-1ff0-2779e7b5e3962d60

Jang, K. L., Lee, S. h., Huh, H. J., \& Chae, J. H. (2015). Influence of the 5-HT3A Receptor Gene Polymorphism and Childhood Sexual Trauma on Central Serotonin Activity. PLoS ONE, 10(12), 1-12. https://doi.org/10.1371/journal.pone.0145269

Jin, M. J, Kim, J. S, Kim, S., Hyun, M. H., \& Lee, S. H. (2018). An Integrated Model of Emotional Problems, Beta Power of Electroencephalography, and Low Frequency of Heart Rate Variability after Childhood Trauma in a Non-Clinical Sample: A Path Analysis Study. Frontiers in psychiatry, 8(314), 1-9. https://doi.org/10.3389/fpsyt.2017.00314

Lackner, C. l., Santesso, D. L., Dywan, J., O.L.D.D., Wade, T. J., \& Segalowitz, S. J. (2018). Adverse childhood experiences are associated with self-regulation and the magnitude of the errorrelated negativity difference. Biological Psychology, 132, 244-251. https://doi.org/10.1016/j.biopsycho.2018.01.006

Lahat, A.,Tang, A, Tanaka, M., Van Lieshout, R. J., MacMillan. H. L., \& Schmidt, L. A. (2018). Longitudinal Associations Among Child Maltreatment, Resting Frontal Electroencephalogram Asymmetry, and Adolescent Shyness. Child Dev, 89 (3), 746757. https://doi.org/10.1111/cdev.13060

Lee, S. H., Park, Y., Jin, M.J., Lee, Y. J., \& Hahn, S. W. (2017). Childhood Trauma Associated with Enhanced High Frequency Band Powers and Induced Subjective Inattention of Adults. Front Behav Neurosci. 11(148), 1-12. https://doi.org/10.3389/ fnbeh.2017.00148

Lemos, M. (2015). La teoría de la alostasis como mecanismo explicativo entre los apegos inseguros y la vulnerabilidad a enfermedades crómicas. Anales de Psicología, 31 (2), 452-461. Recuperado de http:// scielo.isciii.es/scielo.php?script $=$ sci_arttext\&pid $=$ S0212-97282015000200008 
Lobo, I., Portugal, L. C., Figueira, I., Volchan, E., David, I., García Pereira, M., \& De Oliveira, L. (2015). EEG correlates of the severity of posttraumatic stress symptoms: A systematic review of the dimensional PTSD literature. Journal of Affective Disorders, 183, 210-220. https://doi.org/10.1016/j. jad.2015.05.015

Lupien, S. J., McEwen, B. S., Gunnar, M. R., \& Heim, C. (2009). Effects of stress throughout the lifespan on the brain, behaviour and cognition. Natura Reviews Neuroscience, 10(6), 434-445. https://doi. org/10.1038/nrn2639

Mauriera, F. (2018). Principios de neuroeducación física (2. ed.). España: Bubok Publishing SL.

McEwen, B. S., \& Gianaros, P. J. (2011). Plasticidad cerebral inducida por el estrés y la alostasis. Biomedical and life sciences collection Review of Medicine, 65(5), 1-15. Recuperado de https://www.gador.com.ar/ profesionales-detalle/plasticidad-cerebral-inducida-por-el-estres-y-la-alostasis/

McLaughlin, K. A., Fox, N, A., Zeanah, CH, H., \& Nelson, CH. A. (2011). Adverse Rearing Environments and Neural Development in Children: The Development of Frontal Electroencephalogram (EEG) Asymmetry. Biol Psychiatry, 70(11), 1008-1015. https://doi.org/10.1016/j.biopsych.2011.08.006

Meiers G., Nooner, K., De Bellis, M. D., Debnath, R., \& Tang, A. (2020). Alpha EEG asymmetry, childhood maltreatment, and problem behaviors: A pilot home-based study. Child Abuse \& Neglect, 101, 1-11. https://doi.org/10.1016/j.chiabu.2020.104358.

Mesa-Gresa, P., \& Moya-Albiol, L. (2011). Neurobiología del maltrato infantil: el ciclo de la violencia. Revista de Neurología, 52(8), 489-503. https://doi. org/10.33588/rn.5208.2009256

Miskovic, V., Schmidt, L. A., Georgiades, K., Boyle, M., \& MacMillan, H. L. (2009). Stability of resting frontal electroencephalogram (EEG) asymmetry and cardiac vagal tone in adolescent females exposed to child maltreatment. Developmental Psychobiology, 51(6), 474-487. https://doi.org/10.1002/ dev. 20387

Miskovic, V., Schmidt, L. A., Georgiades, K., Boyle, M., \& Macmilla, H. L. (2010). Adolescent females exposed to child maltreatment exhibit atypical EEG coherence and psychiatric impairment: Linking early adversity, the brain, and psychopathology. Development \& Psychopathology, 22(2), 419-432. https://doi.org/10.1017/S0954579410000155

Murphy, F. C., Nimmo-Smith \& Lawrence, A. D. (2003). Functional neuroanatomy of emotions: A metaanalysis. Cognitive, Affective \& Behavioral Neuroscience, 3(3), 207-233. https://doi.org/10.3758/ CABN.3.3.207

Navalta, C. P., McGee, L., \& Underwood, J. (2018). Adverse Childhood Experiences, Brain Development, and Mental Health: A Call for Neurocounseiing. Journal of Mental Health Counseling, 40(3), 266278. https://doi.org/10.17744/mehc.40.3.07

Noll, J. G., Shenk, C. E., \& Putnma, K. T. (2009). Childhood Sexual Abuse and Adolescent Pregnancy: A Meta-Analytic Update. Journal of Pediatric Psychology, 34(4), 366-378. https://doi.org/10.1093/ jpepsy/jsn098

Organización Mundial de la Salud (2013). Plan de acción sobre salud mental 2013-2020. Recuperado de https://apps.who.int/iris/bitstream/handle/10665/97488/9789243506029_spa.pdf;jsess ionid $=670 \mathrm{C} 1 \mathrm{DFF} 13 \mathrm{~F} 210902 \mathrm{~B} 19 \mathrm{EC} 6 \mathrm{D} 0319 \mathrm{~B} 7$ $7 \mathrm{~A}$ ? sequence $=1$

Pando-Orellana, L. A, Vera-García F., \& Lecumberri-Salazar, B. (2013). Estudios de electroencefalografía en pacientes con trastorno de ansiedad. Rev Mex Neuroci, 14(6), 335-340. Recuperado de https:// www.medigraphic.com/pdfs/revmexneu/rmn2013/rmn136f.pdf

Panic, N., Leoncini, E., De Belvis, G., Ricciardi, W., \& Boccia, S. (2013) Evaluation of the Endorsement of the Preferred Reporting Items for Systematic Reviews and Meta-Analysis (PRISMA) Statement on the Quality of Published Systematic Review and Meta-Analyses. PLOS ONE, 8(12), 1-7. https://doi. org/10.1371/journal.pone.0083138

Peltola, M. J., Bakermans-Kranenburg, M. J., Alink, L. R. A., Huffmeijer, R., Biro, S., \& Van Ijzendoorn, M. H. (2014). Resting frontal EEG asymmetry in children: Meta-analyses of the effects of psychosocial risk factors and associations with internalizing and externalizing behavior. Developmental Psychobiology, 56(6), 1377-1389. https://doi.org/10.1002/ dev. 21223 
Pilnik, S.D. (2010). El concepto de alostasis: un paso más allá del estrés y la homeostasis. Rev. Hosp. Ital. B. Aires, 30(1), 7-12. Recuperado de https://www. hospitalitaliano.org.ar/multimedia/archivos/noticias_attachs/47/documentos/6974_7-12-articulo-pilnik.pdf

Racz, F.S., Atylianou, O., Mukli, P., \& Eke, A. (2019). Multifractal and entropy analysis of resting-state electroencephalography reveals spatial organization in local dynamic functional connectivity. Scientific Reports, 9(1). https://doi.org/10.1038/ s41598-019-49726-5

Ramos-Argüelles, F., Morales, G., Egozcue, S., Pabón, R. M., \& Alonso, M. T. (2009). Técnicas básicas de electroencefalografía: principios y aplicaciones clínicas. Anales del Sistema Sanitario de Navarra, 32(3), 69-82. Recuperado de http://scielo.isciii. es/scielo.php? script $=$ sci_arttext\&pid $=$ S1 137 $66272009000600006 \& \operatorname{lng}=\mathrm{es} \& \ln g=\mathrm{es}$.

Redalyc. (2019). ¿Qué es Redalyc.org? Recuperado de https://www.redalyc.org/redalyc/acerca-de/mision.html

Ricardo, J., \& Rueda, W. (2009). Electroencefalograma y potenciales relacionados con eventos en el trastorno obsesivo compulsivo. Salud Mental, 32(2), 173-181. Recuperado de http://www.scielo.org.mx/scielo.php?script $=$ sci_arttext\&pid $=$ S0185-33252009000200010

Sterley, T. L., Howells, F. M., \& Rusell, V.A. (2011). Effects of early life trauma are dependent on genetic predisposition: a rat study. Behavioral and Brain Functions, 7(11), 1-14. https://doi.org/10.1186/17449081-7-11.

Talamillo, T. (2011). Manual básico para enfermeros en electroencefalografía. Enfermería docente, 94, 2933. Recuperado de http://www.sspa.juntadeandalucia.es/servicioandaluzdesalud/huvvsites/default/ files/revistas/ED-094-07.pdf

Tang, A., Lahat, A., Crowley, M. J., Wu, J., \& Schmidt, L. A. (2019). Neurodevelopmental differences to social exclusion: An event-related neural oscillation study of children, adolescents, and adults. Emotion, 19(3), 520-532. https://doi.org/10.1037/ emo0000456

Tang, A., Lahat, A., Miskovic, V., Tanaka, M., MacMillan, H., Van Lieshout, R., \& Schmidt, L. (2018).
Trajectories of Resting Frontal Brain Activity in Predicting Psychopathology in Adolescent Female Exposed to Childhood Maltreatment. Biological Psychiatry, 81(10), 140-276. https://doi. org/10.1016/j.biopsych.2017.02.949

Teicher, M. H., \& Samson, J. A. (2016). Annual Research Review: Enduring neurobiological effects of childhood abuse and neglect. Journal of Child Psychology and Psychiatry and Allied Disciplines, 57(3), 241-266. https://doi.org/10.1111/jcpp.12507

Tejeiro, J. (2008). Electroencefalografía clínica básica. Madrid, España: Viguera

Thompson, M. P., Kingree, J. B., \& Lamis, D. (2019). Associations of adverse childhood experiences and suicidal behaviors in adulthood in a U.S. nationally representative sample. Child: Care, Health \& Development, 45(1), 121-128. https://doi.org/10.1111/ cch. 12617

Torro, N., Fukusima, S. S., \& Aznar-Casanova, J. N. (2008). Models of brain asymmetry in emotional processing. Psychology \& Neuroscience, 1(1), 63-66. https://doi.org/10.3922/j.psns.2008.1.010

Treviño, M., \& Gutiérrez, R. (2007). Las bases celulares de las oscilaciones neuronales. Salud Mental, 30(2), 11-18. Recuperado de http://www.scielo.org.mx/ $\mathrm{pdf} / \mathrm{sm} / \mathrm{v} 30 \mathrm{n} 2 / 0185-3325-\mathrm{sm}-30-02-11 . p d f$

Urrútia, G., \& Bonfill, X. (2010). Declaración PRISMA: una propuesta para mejorar la publicación de revisiones sistemáticas y metaanálisis. Med. Clin, 135(11), 507-511. Recuperado de https:// es.cochrane.org/sites/es.cochrane.org/files/public/ uploads/PRISMA_Spanish.pdf

Witt, A., Brown R. C, Plener, P. L., Brahler E., \& Fegert, J. M. (2017). Maltrato infantil en Alemania: Tasas de prevalencia en la población general. Psiquiatría infantil y adolescente y salud mental, 11(47), 1-9. https://doi.org/10.1186/s13034-017-0185-0

Zare, M., Narayan, M., Lasway, A., Kitsantas, P., Wojtusiak, J., \& Oetjen, C. A. (2018). Influence of adverse Childhood Experiences on Anxiety and Depression in Children Aged 6 to 11 Years. Pediatric Nursing, 44(6), 267-274. Recuperado de https:// conversationsfromwestsalem.files.wordpress. com/2019/01/aces-1.jpeg.pdf 